\title{
Effects of Subcutaneous Interleukin-2 Therapy on CD4 Subsets and In Vitro Cytokine Production in HIV+ Subjects
}

\author{
Paolo De Paoli, ${ }^{\star}$ Stefania Zanussi, ${ }^{\star}$ Cecilia Simonelli, ${ }^{\prime}$ Maria Teresa Bortolin, ${ }^{\star}$ Monica D’Andrea, ${ }^{\star}$ Cinzia Crepaldi, \\ Renato Talamini, $\$$ Manola Comar, "Mauro Giacca," and Umberto Tirelli ${ }^{\ddagger}$ \\ $*$ Department of Microbiology, Immunology, and Virology, ${ }^{\ddagger}$ Department of AIDS Medical Oncology, and ${ }^{\S}$ Department of Epidemiology, \\ Centro di Riferimento Oncologico, IRCCS, 33081 Aviano, Italy; and ${ }^{\|}$Department of Molecular Medicine, International Centre for Genetic \\ Engineering and Biotechnology, 34100 Trieste, Italy
}

\begin{abstract}
HIV infection is characterized by the reduction of the CD4+, CD45RA+, CD26+, and CD28+ lymphocyte subsets and of the in vitro production of IL-2, IL-4, and interferon- $\gamma$; on the contrary, chemokine production is usually increased. These abnormalities are only partially restored by antiretroviral chemotherapy. Therapy with interleukin-2 has been proposed to restore the functions of the immune system, but the mechanisms by which IL-2 exerts its activities are unknown. The aim of this study was to define the effects of rIL-2 administration on CD4+, CD45RA+, $\mathrm{CD} 45 \mathrm{R0}+$, and CD26+ lymphocytes and on the in vitro production of IL-2, IL-4, IL-10, IFN- $\gamma$, RANTES, and SCD30 in HIV + patients.
\end{abstract}

$10 \mathrm{HIV}+$ patients with CD4 cell counts between 200 and 500 cells $/ \mathrm{mm}^{3}$ were treated with six cycles of subcutaneous recombinant IL-2 administration, in combination with zidovudine and didanosine. This therapeutic regimen resulted in a remarkable increase in the number of CD4+ cells and in the prolonged reduction of the levels of viremia. CD45R01 cells were expanded during the first cycle of therapy, while CD45RA+/CD26+ cells predominated after the third cycle. At this time, the in vitro production of IL-2, IL-4, IFN- $\gamma$, and SCD30 were significantly upregulated.

These results demonstrate that rIL-2 in $\mathrm{HIV}+$ patients induces the reconstitution of the CD4/CD45RA lymphocytes subtype. This expanded cell population recovered the ability to produce in vitro IL-2, IL-4, and IFN- $\gamma$. These effects may be beneficial to HIV + patients by improving their immune response to microorganisms or vaccines. (J. Clin. Invest. 1997. 100:2737-2743.) Key words: interleukin-2 injections • HIV infection - HIV viremia - CD4-positive T-lymphocytes $\bullet$ cytokines

\section{Introduction}

In vitro experiments have shown that the natural history of HIV infection may depend on the imbalanced production of

Address correspondence to Dr. Paolo De Paoli, Head, Department of Microbiology, Immunology, and Virology, Centro di Riferimento Oncologico IRCCS, 33081 Aviano, Italy. Phone: 39434 659404; FAX: 39434 659402; E-mail: pdepaoli@ets.it

Received for publication 15 July 1997 and accepted in revised form 26 September 1997.

J. Clin. Invest.

(C) The American Society for Clinical Investigation, Inc. 0021-9738/97/12/2737/07 \$2.00

Volume 100, Number 11, December 1997, 2737-2743

http://www.jci.org certain cytokines secreted by the cells of the immune system (1-4). HIV itself has been suggested to be the major factor that alters the production of cytokines during human infection, by increasing proinflammatory cytokines such as TNF- $\alpha$ or IL-6, and by progressively reducing Th1 cytokines such as IL-2 and IFN- $\gamma$ in favor of Th2 cytokines, namely IL-4 and IL-10 $(1,2)$. The functions of Th1 and Th2 cells, in fact, correlate with the distinctive cytokines they produce. Th1 cells are involved in cell-mediated inflammatory reactions, while Th2 cytokines encourage antibody production $(4,5)$.

Therefore, the persistence and progression of HIV infection could be realized by two mechanisms: first, proinflammatory cytokines increase viral replication; second, the reduction of IL-2 and IFN- $\gamma$ production reduces cellular immunity against the virus. The Th1/Th2 switch theory has been questioned by several authors but, in literature, most studies agree in finding, during HIV disease, a common pathway characterized by reduced IL-2 production $(4,6,7)$.

Interestingly, since IL-2 upregulates in vitro chemokine receptors, a link between IL-2 and chemokines has been recently proposed $(8,9)$. Chemokines are very important molecules acting as chemoattractants for the cells of the immune system (10). T lymphocytes and, in particular, memory $\mathrm{T}$ cells, were shown to respond very efficiently to RANTES and to macrophage inflammatory proteins MIP- $1 \alpha$ and MIP-1 $\beta$ (10). Chemokines have also recently gained great importance in HIV infection due to their antiretroviral activity and to the function of chemokine receptors as entry cofactors for HIV (11-13).

Data in the literature have shown that antiretroviral therapy only partially restores immune function (14) and that cytokine-based immunotherapy may be a very promising approach to a long-lasting reconstitution of the immune system and longer survival (15). Based on the above-mentioned in vitro data, IL-2 has been chosen for in vivo immunotherapy in $\mathrm{HIV}+$ subjects, and some studies have shown that it induces a recovery of CD4 cells without increasing viral load and without significant clinical side effects (15-19). Little data on the effects of IL-2 administration in HIV+ patients on the functions of the immune system are available. In particular, it is not known how the CD4 cell subsets expressing important functional antigens such as CD45 isoforms, CD26, and CD28 respond to IL-2 therapy, or how cytokine production is modified by IL-2 administration. Since issues concerning the origin and immunological function of the expanded CD4+ cells during IL-2 therapy are relevant to further progress, we undertook a study to clarify these questions in a cohort of HIV+ patients treated for 6 mo with subcutaneous IL-2 plus antiretroviral therapy.

The aim of this study was to investigate the capacity of subcutaneous IL-2 to modify some selected aspects of the immune system; in particular, we wanted to know whether (a) IL-2 in- 
duces a nonselective or rather the preferential expansion of phenotypically defined CD4 subsets, and (b) IL-2 modifies the imbalance of in vitro cytokine and chemokine production observed in HIV disease.

The data obtained indicate that subcutaneous IL-2 administration is characterized by rapid changes in the phenotype of the expanded CD4 cells and that a peculiar phenotypic profile coupled to cellular functional recovery emerges after 6 mo of therapy.

\section{Methods}

Patients. $10 \mathrm{HIV}$-infected patients (ELISA and Western blot positive) with CD4 counts $200-500$ cells $/ \mathrm{mmc}$, stage A2, B2 according to CDC 1993 classification (20), and without previous antiretroviral treatment were consecutively enrolled to receive AZT (250-mg bid) and DDI (100-mg bid below $50 \mathrm{~kg}, 200-\mathrm{mg}$ bid between 50 and $75 \mathrm{~kg}$, and 300-mg bid above $75 \mathrm{~kg}$ body wt) and self subcutaneous administration of 6 MUI of IL-2 (Chiron Corp., Emeryville, CA): day 1-5 and $8-12$ of a 28 -d cycle for six cycles. Patients were evaluated for hematological, pancreatic, hepatic, and renal functions every $2 \mathrm{wk}$. Immunological and virological parameters were evaluated at times 0,2 , 4 , and 12 (end of cycle 3), and 24 (end of therapy) wk.

Six HIV+ patients with the same inclusion characteristics and treated with the same antiretroviral protocol, but without IL-2, were also included in the study. The clinical results of this protocol will be detailed elsewhere (Simonelli, C., P. De Paoli, S. Zanussi, and U. Tirelli, manuscript in preparation). As controls, we included 15 healthy donors of the same age group of the patients.

Lymphocyte phenotyping. Anticoagulated peripheral whole blood was mixed with the following monoclonal antibodies, alone or in combinations: CD4-FITC, CD8-FITC (Ortho D.S., Milan, Italy), CD26-PE, CD25-PE, CD28-PE (Becton Dickinson \& Co., Milan, Italy); a CD4-PE/CD30-FITC (Dako, Milan, Italy) combination has also been used. Triple immunofluorescence assay was performed using CD4-Cy5 (Dako)/CD45RA-PE (Coulter, Milan, Italy)/CD45R0FITC (Dako). After incubation, cells were fixed with Immunoprep (Coulter), and fluorescence was measured in an EPICS XL flow cytometer (Coulter). Appropriate isotypic negative controls were run in parallel.

Absolute lymphocyte counts were calculated by standard haemocytometric technique.

Cell preparation and in vitro culture. Peripheral blood mononuclear cells were separated by centrifugation on Ficoll (Nycomed, Milan, Italy); cells were recovered, washed, and incubated in RPMI 1640, $10 \%$ FCS, at $3 \times 10^{6}$ cells $/ \mathrm{ml}$ concentration, in the presence or absence of $1 \%$ PHA (Gibco Laboratories, Milan, Italy), as previously described (21). Culture supernatants were harvested after $48 \mathrm{~h}$ of incubation, centrifuged, filtered, and frozen until cytokine or sCD30 measurements were performed.

In vitro cytokine and $S C D 30$ production. Cytokine production in supernatants was measured by commercial ELISA assays (IL-2, IL-4, IL-10, IFN- $\gamma$; Biosource, CELBIO, Milan, Italy; RANTES; R \& D Systems, Inc., Milan, Italy). Briefly, the appropriate culture supernatant aliquots were added to anticytokine antibody-coated microtiter wells. After incubation and washings, a second monoclonal antibody against the assay cytokine and labeled with biotin was added. Horseradish peroxidase-labeled streptavidin and the chromogen were added and the absorbance read at $450 \mathrm{~nm}$. The sample cytokine concentrations were calculated by interpolating experimental data with the absorbance of a standard curve. Results were then expressed in picograms per milliliter.

Soluble CD30 concentration was determined by a mouse monoclonal antibody sandwich assay (Dako) using simultaneous incubation of samples and peroxidase conjugate in antibody-coated microwells. Coating and conjugate monoclonal antibodies are noncompetitive and react with different epitopes of CD30. A chromogenic substrate was added to the wells and the absorbance measured at $450 \mathrm{~nm}$. A standard curve was prepared from six CD30 calibrators and the concentration of sCD30 in specimens was determined by interpolation; the results obtained were expressed as units per milliliter.

Virus plasma concentration. The levels of viremia were evaluated by competitive reverse transcription and polymerase chain reaction, as described previously $(22,23)$. Briefly, viral RNA was extracted from the virion pellet after ultracentrifugation $(40,000 \mathrm{rpm}$ on a TST 55.5 rotor [Kontron, Milan, Italy] for $60 \mathrm{~min}$ at $4^{\circ} \mathrm{C}$ ) of $5 \mathrm{ml}$ of plasma. For each quantification, fixed aliquots of sample RNA were mixed with $3 \mu l$ of opportunely diluted competitor RNA and submitted to competitive reverse transcription-PCR. Cycling profiles were as follows: denaturation at $95^{\circ} \mathrm{C}$ for $30 \mathrm{~s}$, annealing at $60^{\circ} \mathrm{C}$ for $30 \mathrm{~s}$, and extension at $72^{\circ} \mathrm{C}$ for $72 \mathrm{~s}$.

After amplification, $20 \mu \mathrm{l}$ of each PCR product was resolved on $8 \%$ nondenaturing polyacrylamide gels, visualized under ultraviolet light after ethidium bromide staining, and photographed. Quantification of the amplification products was obtained by densitometric scanning of the ethidium bromide-stained gels.

Statistical analysis. Since the variables under study were not normally distributed, nonparametric statistical tests were chosen. The Mann-Whitney test was used to compare the distributions of variables between two groups, and Wilcoxon rank sum test was used to analyze paired values in the same group (24). Results were considered statistically significant when $P \leq 0.05$ (two tails).

\section{Results}

CD4 and CD8 values, and viremia before and after therapy. The CD4 and CD8 cell counts and the levels of plasma viremia of $10 \mathrm{HIV}+$ patients enrolled to received rIL-2 plus AZT/DDI (group I), of $6 \mathrm{HIV}+$ subjects treated only with AZT/DDI (group II), and the HIV controls (group III) are shown in Table I. Both groups I and II had reduced CD4 levels before therapy; the data obtained in the two groups after therapy demonstrate that in group I (IL-2-treated patients), a statistically significant increase of CD4 percentages $(P \leq 0.01$ vs. group II) and absolute numbers $(P \leq 0.05$ vs. group II) was obtained, while CD8 cell values gave similar results. HIV plasma viremia was not increased in IL-2-treated HIV subjects in comparison with patients treated with antiretrovirals alone.

The clinical and virological results of this study will be detailed elsewhere (Simonelli et al., manuscript in preparation).

CD4 subsets before and after therapy. CD4 subsets in response to rIL-2 plus AZT/DDI or to AZT/DDI alone were evaluated before and after therapy (Table II). Two important patterns of changes were seen in group I as compared with group II patients. First, both CD4/CD45RA+ and 45R0+ cells are reduced in HIV + patients before therapy, but only rIL2 -treated subjects show a recovery of $45 \mathrm{RA}+$ cells after therapy $(16.7 \pm 6 \%$ in group I vs. $10 \pm 6 \%$ in group II, $P \leq 0.05)$; second, a similar change is observed for CD4/CD26+ cells $(13.1 \pm 7 \%$ in group I vs. $6.2 \pm 3 \%$ in group II, $P \leq 0.05)$. The percentage of CD4/CD25+ lymphocytes before therapy was $1.6 \pm 0.7$ in group I and $1.6 \pm 0.9$ in group II. At $24 \mathrm{wk}$, IL-2 treatment induces a significant increase in CD4/CD25+ lymphocytes $(5.9 \pm 2 \%$ in group I vs. $2.4 \pm 0.5 \%$ in group II, $P \leq$ 0.01). No significant changes are seen considering CD4/ CD28+ cells and no CD4/CD30+ lymphocytes could be detected before or after therapy (data not shown).

In conclusion, these data demonstrate that the CD4+/ CD45RA+/CD26+ subset is preferentially reconstituted by IL-2 administration in HIV+ patients. 


\begin{tabular}{|c|c|c|c|c|c|c|c|c|c|c|}
\hline & \multicolumn{4}{|c|}{$\mathrm{CD} 4$} & \multicolumn{4}{|c|}{$\mathrm{CD} 8$} & \multirow{2}{*}{\multicolumn{2}{|c|}{ Viremia }} \\
\hline & \multicolumn{2}{|c|}{ Percentage } & \multicolumn{2}{|c|}{ Absolute numbers } & \multicolumn{2}{|c|}{ Percentage } & \multicolumn{2}{|c|}{ Absolute numbers } & & \\
\hline & $t=0 w k$ & $t=24 w k$ & $t=0 w k$ & $t=24 w k$ & $t=0 w k$ & $t=24 w k$ & $t=0 w k$ & $t=24 w k$ & $t=0 w k$ & $t=24 w k$ \\
\hline HIV + IL-2 group I & $19.9 \pm 5$ & $30.6 \pm 7 * *$ & $331 \pm 71$ & $652 \pm 194 *$ & $57.7 \pm 8$ & $48 \pm 8$ & $1078 \pm 640$ & $1071 \pm 360$ & $3.6 \pm 4.9 \times 10^{4}$ & $\begin{array}{c}<500 \\
9 / 10\end{array}$ \\
\hline HIV group II & $18.8 \pm 10$ & $21.6 \pm 7$ & $330 \pm 115$ & $392 \pm 136$ & $59.6 \pm 7$ & $57.6 \pm 7$ & $1154 \pm 449$ & $1075 \pm 304$ & $4.1 \pm 1 \times 10^{5}$ & $\begin{array}{c}<500 \\
4 / 6\end{array}$ \\
\hline Controls group III & $40.6 \pm 10 * *$ & & $720 \pm 160 *$ & & $33 \pm 12 *$ & & $555 \pm 340 *$ & & & \\
\hline
\end{tabular}

Percentage and absolute numbers (cells $\left./ \mathrm{mm}^{3}\right)$ of CD4 and CD8 subsets and HIV plasma viremia (RNA copies/ml) of HIV+ patients treated with antiretrovirals plus IL-2 (group I), HIV+ patients treated with antiretrovirals alone (group II) before $(\mathrm{t}=0$ wk) and after $(\mathrm{t}=24 \mathrm{wk})$ treatment; $* P \leq$ $0.05 ;{ }^{*} P \leq 0.01 ; P$ refers to statistical significance of group I vs. group II. Group III includes healthy donors.

Pattern of cytokine and sCD30 production before and after therapy. In all the experiments, spontaneous cytokine production was $<10 \%$ of the values obtained from stimulated cultures. The data in Table III show stimulated minus spontaneous cytokine production (picograms per milliliter).

HIV infection induces a rather nonselective pattern of reduction of in vitro cytokine production, since IL-2, IL-4, and IL-10 are significantly reduced before therapy compared with controls; rIL-2 administration is able to increase the production of all cytokines except IL-10. Although the comparison between groups I and II at the end of the therapy reveals statistically significant differences for IL-2 and IL-4 production ( $P \leq 0.05$ for both cytokines), a greater increase in group I has also been found for IFN- $\gamma$. In the latter case, however, the difference is not statistically significant because of the low number of patients in the study groups. Furthermore, the sequential measurements of cytokine production demonstrate that IL-2 induces the maximum increase of in vitro IFN- $\gamma$ production after 12 wk of therapy (see Fig. 2).

The in vitro production of RANTES is increased at baseline in both groups of HIV+ patients as compared with controls. After $24 \mathrm{wk}$, RANTES production is similar in the two groups of HIV+ subjects, irrespective of IL-2 administration.

Soluble CD30 production (data not shown) was not increased at baseline in HIV+ patients compared with controls $(19 \pm 25 \mathrm{U} / \mathrm{ml}$ in group I, $7 \pm 6 \mathrm{U} / \mathrm{ml}$ in group $\mathrm{II}, 18 \pm 8 \mathrm{U} / \mathrm{ml}$ in controls). After $24 \mathrm{wk}$, sCD30 levels were $24 \pm 20 \mathrm{U} / \mathrm{ml}$ in group I and $11 \pm 7 \mathrm{U} / \mathrm{ml}$ in group II $(P \leq 0.01)$.
Based on these results, we can conclude that IL-2 upregulates the in vitro production of all the cytokines we have studied, with the exception of IL-10.

Sequential changes of CD4 subsets during IL-2 administration. Next, we have investigated how CD4 subsets behave in the course of IL-2 therapy. The data obtained at time 0, 2, 4 (end of cycle 1), 12 (end of cycle 3), and 24 (end of therapy) wk are reported in Fig. 1. The percentage and absolute numbers of CD 4 cells are increased after 2 wk of therapy and remain elevated for the entire period of observation. The composition of the CD4 subset varies according to time, because the percentage of CD4+/CD45R0+ cells is increased soon (week 2, $P \leq 0.01$ ), while the increase of the percentages of naive $\mathrm{CD} 4+$ / CD45RA+ and of CD4+/CD26+ cells reach significance only after 12 wk of therapy $(P \leq 0.01)$. In particular, the ratio between the percentage of RA+ and R0+ CD4 cells is $1: 1$ at time $0,0.57$ at $2,1.05$ at $4,1.11$ at 12 , and 1.45 at 24 wk, demonstrating that long term IL-2 administration preferentially expands the pool of naive CD4 cells.

The effects of IL-2 treatment in HIV+ patients may be different from those obtained in the HIV-negative population because, in the course of HIV disease, precursor cells may have been eliminated or altered; our data suggest that the recovery of CD4 cells induced by IL-2 in HIV+ patients is a dynamic process because $\mathrm{CD} 45 \mathrm{R} 0+$ memory cells increase at early times, while CD45RA + naive cells constitute the long term expanded CD4 population.

Sequential changes of cytokine and sCD30 production dur-

Table II. CD4 Subsets before and after Therapy

\begin{tabular}{|c|c|c|c|c|c|c|c|c|c|c|c|c|}
\hline & \multicolumn{4}{|c|}{$\mathrm{CD} 4 / \mathrm{CD} 45 \mathrm{RA}$} & \multicolumn{4}{|c|}{$\mathrm{CD} 4 / \mathrm{CD} 45 \mathrm{RO}$} & \multicolumn{4}{|c|}{$\mathrm{CD} 4 / \mathrm{CD} 26$} \\
\hline & \multicolumn{2}{|c|}{ Percentage } & \multicolumn{2}{|c|}{ Absolute numbers } & \multicolumn{2}{|c|}{ Percentage } & \multicolumn{2}{|c|}{ Absolute numbers } & \multicolumn{2}{|c|}{ Percentage } & \multicolumn{2}{|c|}{ Absolute numbers } \\
\hline & $t=0 w k$ & $t=24 w k$ & $t=0 w k$ & $t=24 w k$ & $t=0 w k$ & $t=24 w k$ & $t=0 w k$ & $t=24 w k$ & $t=0 w k$ & $t=24 w k$ & $t=0 w k$ & $t=24 w k$ \\
\hline HIV + IL-2 group I & $9.3 \pm 5$ & $16.7 \pm 6^{*}$ & $153 \pm 68$ & $337 \pm 186^{*}$ & $8.1 \pm 3$ & $11.5 \pm 4$ & $141 \pm 37$ & $247 \pm 138$ & $7 \pm 4$ & $13.1 \pm 7 *$ & $106 \pm 70$ & $259 \pm 101 *$ \\
\hline $\mathrm{HIV}$ + group II & $9.7 \pm 5$ & $10 \pm 6$ & $167 \pm 7$ & $160 \pm 71$ & $7.6 \pm 4$ & $8.6 \pm 3$ & $194 \pm 58$ & $150 \pm 53$ & $4.8 \pm 3$ & $6.2 \pm 3$ & $82 \pm 42$ & $118 \pm 69$ \\
\hline Controls group III & $18.3 \pm 6^{* *}$ & & $275 \pm 112 *$ & & $16.8 \pm 7 * *$ & & $252 \pm 114 *$ & & $12.8 \pm 5 * *$ & & $196 \pm 71 *$ & \\
\hline
\end{tabular}

Percentage and absolute numbers (cells $/ \mathrm{mm}^{3}$ ) of CD45RA, CD45R0, and CD26 positive cells within the CD4 subset in HIV + patients treated with antiretrovirals plus IL-2 (group I), or with antiretrovirals alone (group II) before $(\mathrm{t}=0 \mathrm{wk})$ and after $(\mathrm{t}=24 \mathrm{wk})$ treatment; $* P \leq 0.05 ; * * P \leq 0.01, P$ refers to statistical significance of group I vs. group II. Group III includes healthy donors. 
Table III. Cytokine and sCD30 Production before and after Therapy

\begin{tabular}{|c|c|c|c|c|c|c|c|c|c|c|}
\hline & \multicolumn{2}{|c|}{ IL-2 } & \multicolumn{2}{|c|}{ IL-4 } & \multicolumn{2}{|c|}{ IL-10 } & \multicolumn{2}{|c|}{ IFN- $\gamma$} & \multicolumn{2}{|c|}{ RANTES } \\
\hline & $t=0 w k$ & $t=24 w k$ & $t=0 w k$ & $t=24 w k$ & $t=0 w k$ & $t=24 w k$ & $t=0 w k$ & $t=24 w k$ & $t=0 w k$ & $t=24 w k$ \\
\hline HIV + IL-2 group I & $41 \pm 47$ & $138 \pm 167^{*}$ & $9 \pm 5$ & $112 \pm 81^{*}$ & $647 \pm 370$ & $631 \pm 319$ & $7059 \pm 5790$ & $28730 \pm 22400$ & $5224 \pm 5022$ & $9690 \pm 5100$ \\
\hline HIV group II & $43 \pm 26$ & $62 \pm 41$ & $3.5 \pm 5$ & $29 \pm 42$ & $360 \pm 116$ & $542 \pm 305$ & $11890 \pm 11200$ & $7520 \pm 6100$ & $6690 \pm 4510$ & $11510 \pm 9970$ \\
\hline Controls group III & $320 \pm 190 * *$ & & $34 \pm 39 *$ & & $998 \pm 250^{*}$ & & $9850 \pm 9500$ & & $2830 \pm 9500$ & \\
\hline
\end{tabular}

In vitro cytokine production (expressed as picograms per milliliter) from PHA-stimulated peripheral blood mononuclear cells from HIV+ patients treated with antiretrovirals plus IL-2 (group I) or antiretrovirals alone (group II); $* P \leq 0.05 ; * * P \leq 0.01, P$ refers to statistical significance in group I vs. group II. Group III is composed of healthy controls. Cytokine production from nonstimulated cells was $<10 \%$ and was subtracted from stimulated values.

ing IL-2 administration. The results of cytokine production during IL-2 therapy are reported in the panels of Fig. 2. The restoration of a normal cytokine production profile is quite long, requiring $12 \mathrm{wk}$ of this schedule of therapy with IL-2. At this time point, IL-2, IL-4, and IFN- $\gamma$ production are significantly increased as compared with time 0 , while no effects on
IL-10 production can be noticed. On the contrary, RANTES production is rapidly upregulated, but its production declines at 12 and 24 wk. Similarly, sCD30 values are increased after 2 and $4 \mathrm{wk}$, with the tendency of return to baseline values at the end of therapy.

These data suggest that, in HIV+ patients, IL-2 acts in vivo
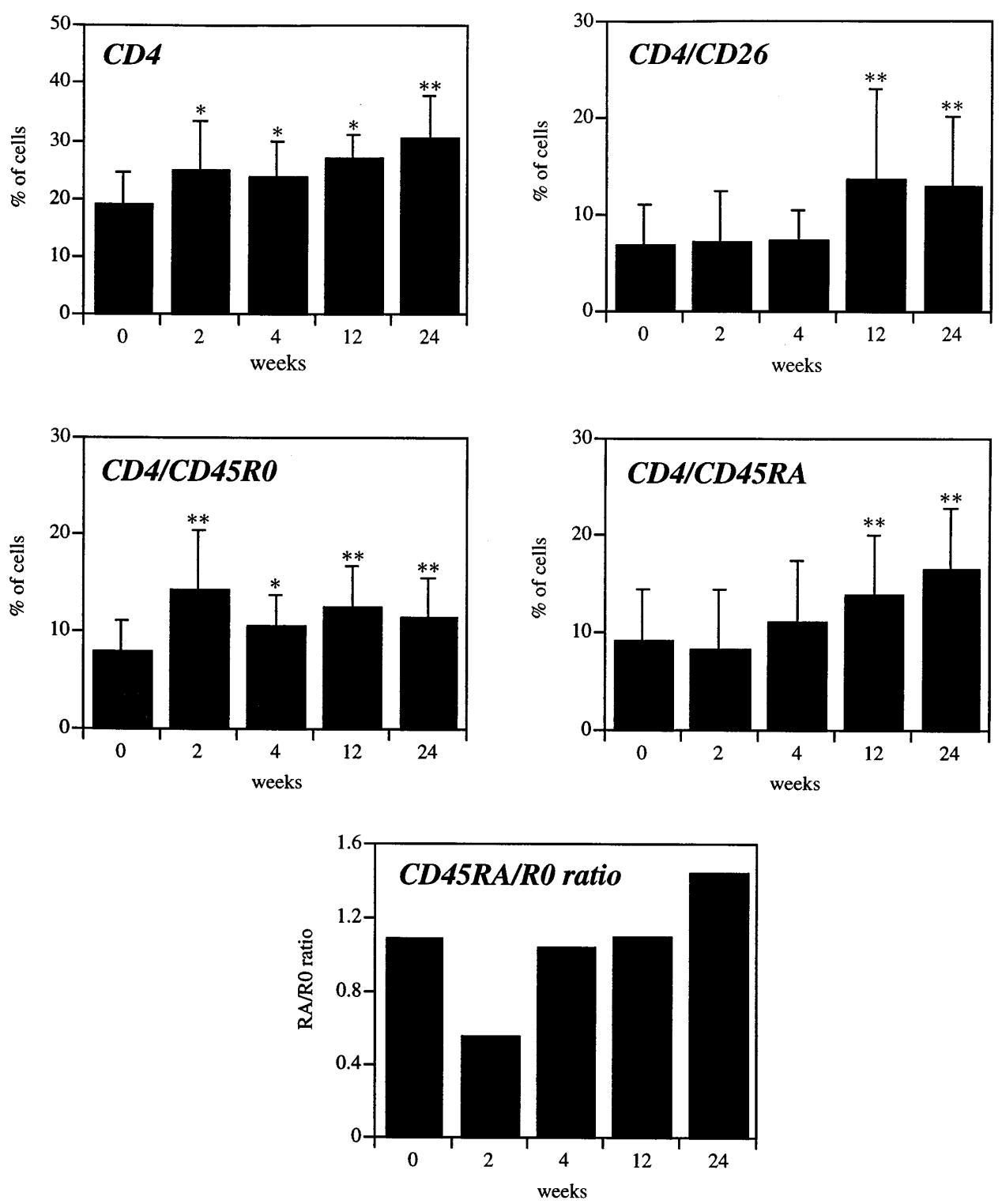

Figure 1. CD4 lymphocyte subsets during IL-2 administration. The percentages (bars) and standard deviations (lines) of CD4, CD4/45RA, CD4/CD45R0, CD4/CD26 cell subsets, and the CD45RA/CD45R0 ratio before (time 0 ) and at different times after beginning of IL-2 therapy are shown. Asterisks indicate statistical significance vs. the pretreatment levels. $* P \leq 0.05 ; * * P \leq$ 0.01 . 

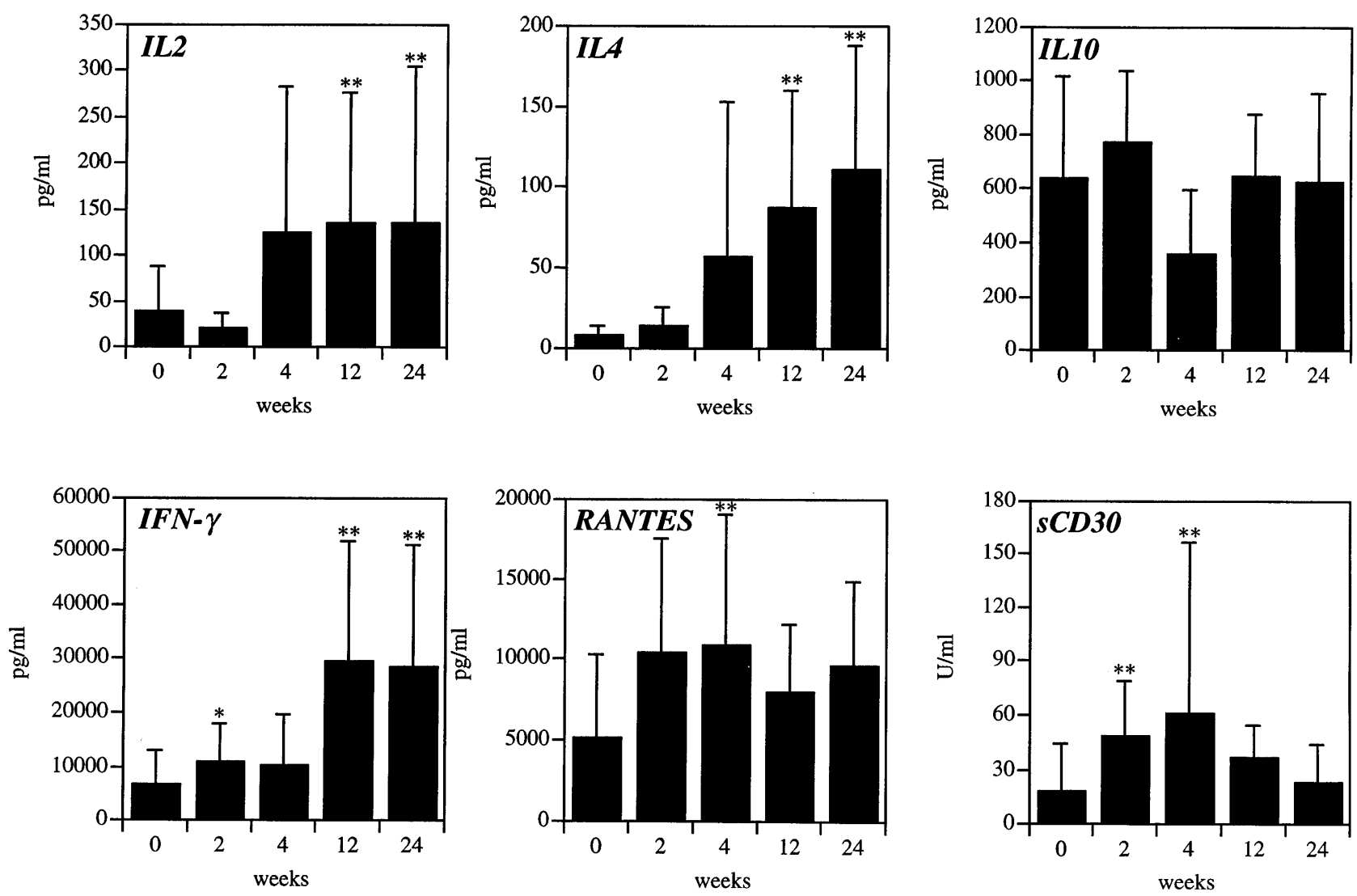

Figure 2. In vitro cytokine and sCD30 production during IL-2 administration. The graphs show the levels of cytokine $(\mathrm{pg} / \mathrm{ml})$ and sCD30 $(U / \mathrm{ml})$ production by PHA-stimulated peripheral blood mononuclear cells of HIV+ patients before (time 0 ) and during IL-2 administration. The level of cytokine production from nonstimulated cell cultures was always $<10 \%$ of the respective values obtained from stimulated cells; the plotted values are the differences between the two measurements. Asterisks indicate statistical significance vs. the pretreatment levels. $* P \leq 0.05 ; * * P \leq$ 0.01 .

as a pleiotropic cytokine, exerting its maximum activity after $12 \mathrm{wk}$ of in vivo administration.

\section{Discussion}

HIV infection produces a progressive reduction of the CD4 pool and a very precocious functional impairment of this subset. The most striking abnormalities seen are the reduction of the naive pool and the inability to produce in vitro cytokines such as IL-2, IFN- $\gamma$ and others $(1-3,25,26)$. In vivo and in vitro data demonstrate also that chemokine production is generally increased in HIV subjects $(27,28)$.

Antiretroviral chemotherapy is able to increase for a short time CD4 counts, but its effects on cellular functions are very limited (14). Thus, in addition to antiretroviral treatment, the reconstitution of the immune function represents the primary therapeutic goal of HIV infection. IL-2 appears to be a good candidate for this purpose since IL-2 depletion is probably one of the most striking defects in HIV patients. In addition, it should be considered that a considerable therapeutic experience on the beneficial effects of this cytokine on the immune function has been obtained in treating cancer patients $(29,30)$. One theoretical concern of IL-2 treatment in HIV+ patients is related to its ability to induce cellular activation. HIV infection, in fact, is accompanied by a chronic activation of the immune system; such chronic activation has been proposed as a mechanism by which continued virus replication is facilitated $(3,15)$.

Some clinical trials with IL-2 have been performed in HIV patients. Kovacs et al. (18) and Davey et al. (31) have shown that intermittent administration of high doses of IL-2 i.v. (18 $\mathrm{MUI} / \mathrm{d}$ ) and/or s.c. (3-18 MUI/d) gave a significant increase of CD 4 counts in asymptomatic patients. The side effects reported in earlier studies (32), like capillary leak, severe flu-like symptoms, and increased viral load were less relevant in subsequent studies, especially when a subcutaneous route of administration has been used (31).

In our experience, intermediate doses of subcutaneous IL-2 (6 MUI/d, days 1-5 and 8-12 of a 28 -d cycle, for a total of six cycles) were well tolerated as an outpatient regimen: only mild constitutional effects were observed and all the treated patients performed their usual daily activities. In conclusion, the feasibility of our therapeutic schedule was superimposable with the other trials using subcutaneous IL-2 administration $(17,31)$. When the effects of IL-2 treatment on CD4 cell counts are analyzed, the use of ultra low doses of IL-2 was unable to obtain a statistically significant increase of CD4 cell counts (17); on the contrary, our therapeutic regimen and that described by Kovacs et al. (18) produce a significant increase in these cell numbers. Concerning the possible enhancing effects of IL-2 administration on HIV viremia, only two of our patients showed a transient increase of viral load after the first 
and third cycle; these results are consistent with previous observations $(18,31)$.

None of the above mentioned studies on IL-2 administration in HIV + patients, however, investigated the effects of IL-2 on CD4 cell phenotype and in vitro cytokine production. The knowledge of how IL-2 influences the functions of the immune system and the length of its action are important parameters for a better clinical understanding of its efficacy. For these reasons, we have evaluated the immunological and virological effects of subcutaneous IL-2 therapy in a group of HIV subjects.

The results obtained by this work show that IL-2 produces an early increase in CD4 cells that is still present after $24 \mathrm{wk}$ of therapy. At this time point, IL-2-treated subjects demonstrate a reconstitution of CD45RA,$+ \mathrm{CD} 26+$ lymphocytes, a subset that is partially lost during the natural course of HIV infection $(26,33)$. In addition, IL-2 administration also induces, after 12 wk of therapy, a substantial reconstitution of the peripheral blood lymphocytes that produce IL-2, IL-4, and IFN- $\gamma$.

Although the use of CD45 isoforms does not fully define the naive/memory pool, this characterization of the CD4 subset has been widely used for clinical and research purposes (19, 34). In fact, Connors et al. (19) have recently shown that CD4/ CD45RA+ cells are recovered after IL-2 infusion in HIV+ patients. Here, we demonstrate that the balance between CD4/ CD45RA + and CD4/CD45R0+ cells is a dynamic event: the first cycle of IL-2 preferentially expands the CD4/CD45R0+ memory cells, while a CD4/CD45RA+ population becomes predominant after $12 \mathrm{wk}$ of therapy. We cannot formally exclude the theory that the CD4/CD45R0 expansion in the peripheral blood of patients during IL-2 administration depends on the redistribution of cells from lymphoid organs. This explanation, however, is unlikely to be correct, considering that in vivo data have demonstrated that there is a $>10$-fold increase in the percentage of lymphocytes that proliferate in response to IL-2 (16). Furthermore, although the CD45RA/ CD45R0 transition is not an irreversible event (35), CD45RA+ naive cells have been generally considered to be the precursors of memory cells because they predominate at the thymic level and, once matured, convert to a CD45R0+ phenotype upon in vitro mitogen stimulation and in response to IL-2 (36). Therefore, we favor the hypothesis that in vivo IL-2 administration drives CD4 lymphocytes to a memory phenotype. As shown by in vitro experiments, the continuous stimulation probably induces these cells to die preferentially (activation-associated lymphocyte death, AALD; reference 37). The naive CD4 cells are more reluctant to activate signals, but possess longer telomeric restriction fragments, indicating higher replicative potential (38), and are more resistant to AALD (37); for these reasons they may be preferentially expanded in our patients after long term IL-2 administration.

Experimental data have recently demonstrated differences in the rate of viral production and susceptibility between naive and memory subpopulations (39). The ability of CD4/ CD45RA + cells to replicate HIV-1, in fact, appear to be restricted by a labile postfusion complex that requires cellular activation signals beyond that provided by IL-2 alone for productive infection (39). We herein show that preservation of the naive pool is operative in vivo in $\mathrm{HIV}+$ patients undergoing cytokine administration. The limited capacity of naive cells to sustain HIV replication and their greater replicative potential, indicating a major capacity to sustain levels necessary for intact immune competence (38), are strong arguments suggest- ing that their IL-2-induced expansion may be considered a very favorable event in HIV+ subjects.

We have also observed that the CD4 increase during subcutaneous IL-2 administration was reflected by improved cellular immune functions, namely an increased capacity to produce cytokines in vitro. IL-2 acts in vivo as a pleiotropic cytokine, inducing the synthesis of IL-2 itself, IL-4, and IFN- $\gamma$. The effects on cytokine production are slow but persistent, appearing after 12 and still present after $24 \mathrm{wk}$ of therapy. It is very difficult to reconcile these data with the simple notion that a Th1/Th2 cell switch parallels the progression of HIV infection. On the contrary, the cellular and cytokine interplay that are modulated in vivo by the therapy with IL-2 appear far more complex, due to the following considerations. First, the $\mathrm{HIV}+$ patients in our study show before therapy a nonselective pattern of reduced cytokine production, rather than a selective Th1-like, IL-2, or IFN- $\gamma$ defect. Second, while the Th1or Th2-inducing capacity of several soluble mediators is well known $(4,5)$, the importance of IL-2 in priming in vitro T cells to develop into Th1 or Th2 cells is made difficult by the importance of IL-2 itself in growth and survival of cells in the course of priming culture (5). The in vivo upregulation of IL-4 production by IL-2 is not, however, surprising since Ben-Sasson et al. (40) have shown that IL-2 is required for in vitro IL-4 production by $\mathrm{T}$ cells from naive donors. Third, IL-2 administration in our patients is followed by pleiotropic effects on the immune system, including an increase of Th1 (IL-2, IFN- $\gamma$, CD26) $(4,5,41,42)$, as well as Th2 (IL-4, sCD30)-associated immune markers $(4,5,43,44)$. Fourth, it is conceivable that in vivo IL-2 administration during HIV infection might allow only a time-limited modulation of the cytokine response without permanently altering the $\mathrm{T}$ cells.

The serum levels of RANTES are elevated in HIV+ patients compared with healthy controls as a part of the immunological response to viral infections $(11,27,45)$ and in vitro experiments have shown that IL-2 upregulates chemokine production by lymphocytes (11). Similarly, in vivo IL-2 administration to HIV + subjects is able to induce a rapid upregulation of in vitro RANTES production, but results obtained after 24 wk are similar in IL-2-treated patients and in subjects treated with antiretrovirals alone. Because of the sensitivity of memory cells to chemokine actions, it is possible that these molecules may act as a cofactor for the expansion of CD4/ CD45R0+ cells during the first cycle of IL-2 administration, but our data suggest that, in long lasting IL-2 therapy, their role, if any, is probably limited.

Although previous studies have shown that the CD4 T cell repertoire is not fully restored by immune-based therapies (19), the reconstitution of CD4 lymphocytes that are similar for phenotype and cytokine production to the corresponding cells in healthy controls is a favorable event obtained by IL-2 therapy. This therapy could have an important impact on HIV disease, not only because the phenotype of the long term CD4+ expanded cells corresponds to that of cells having a restricted pattern of HIV replication in vitro, but also by temporarily influencing host immune response to microorganisms or to appropriate vaccines.

\section{Acknowledgments}

This paper has been supported by ISS grants. 


\section{References}

1. Clerici, M., and G. Shearer. 1994. Th1-Th2 hypothesis in HIV infection: new insights. Immunol. Today. 15:575-581.

2. Clerici, M., C. Balotta, A. Salvaggio, C. Riva, D. Trabattoni, L. Papagno, A. Berlusconi, S. Rusconi, M.L.Villa, M. Moroni, and M. Galli. 1996. Human Immunodeficiency Virus (HIV) phenotype and interleukin-2/interleukin-10 ratio are associated markers of protection and progression in HIV infection. Blood. 2:574-579.

3. Rosemberg, L.F., and A.S. Fauci. 1990. Immunopathogenetic mechanisms of HIV infection: cytokine induction of HIV expression. Immunol. Today. 11:176-180.

4. Romagnani, S., E. Maggio, and G. Del Prete. 1994. An alternative view of the Th1/Th2 switch hypothesis in HIV infection. AIDS Res. Hum. Retroviruses. 10:3-9.

5. Mosmann, T.R., and S. Subash. 1996. The expanding universe of T-cell subsets: Th1, Th2 and more. Immunol. Today. 17:138-146.

6. Fan, J., H.Z. Bass, and J.L. Fahey. 1993. Elevated IFN- $\gamma$ and decreased IL-2 gene expression are associated with HIV infection. J. Immunol. 151:50315040 .

7. Adachi, Y., N. Oyaizu, S. Than, T.W. McCloskey, and S. Pahwa. 1996. IL-2 rescues in vitro lymphocyte apoptosis in patients with HIV infection. J. Immunol. 157:4184-4193.

8. Loetscher, P., M. Seitz, M. Baggiolini, and B. Moser. 1996. Interleukin-2 regulates $\mathrm{CC}$ chemokine receptor expression and chemotactic responsiveness in T lymphocytes. J. Exp. Med. 184:569-577.

9. Wu, L., W.A. Paxton, N. Kassam, N. Ruffing, J.B. Rottman, N. Sullivan, H. Choe, J. Sodroski, W. Newman, R.A. Koup, and C.R. Mackay. 1997. CCR5 levels and expression pattern correlate with infectability by macrophage-tropic HIV-1, in vitro. J. Exp. Med. 185:1681-1691.

10. Premack, B.A., and T.J. Schall. 1996. Chemokine receptors: gateways to inflammation and infection. Nat. Med. 2:1174-1178.

11. Cocchi, F., A.L. DeVico, A. Garzino-Demo, S.K. Arya, R.C. Gallo, and P. Lusso. 1995. Identification of RANTES, MIP-1, and MIP-1 as the major HIV-suppressive factors produced by CD8+ T cells. Science. 270:1811-1816.

12. D'Souza, M.P., and V.A. Harden. 1996. Chemokines and HIV-1 second receptors. Nat. Med. 2:1293-1300.

13. Moore, J.P. 1997. Coreceptors: implications for HIV pathogenesis and therapy. Science. 276:51-52.

14. Clerici, M., E. Roilides, K.M. Butler, L. DePalma, D. Venzon, G.N. Shearer, and P.A. Pizzo. 1992. Changes in T-helper cell function in human immunodeficiency virus-infected children during didanosine therapy as a measure of antiretroviral activity. Blood. 80:2196-2202.

15. Emery, S., and H.C. Lane. 1996. Immune-based therapies in HIV infection: recent developments. AIDS (Lond.). 10:159-163.

16. Teppler, H., G. Kaplan, K.A. Smith, A.L. Montana, P. Meyn, and Z.A. Cohn. 1993. Prolonged immunostimulatory effect of low-dose polyethylene glycol interleukin 2 in patients with human immunodeficiency virus type 1 infection. J. Exp. Med. 177:483-492.

17. Leef Jacobson, E., F. Pilaro, and K.A. Smith. 1996. Rational interleukin 2 therapy for HIV positive individuals: daily low doses enhance immune function without toxicity. Proc. Natl. Acad. Sci. USA. 93:10405-10410.

18. Kovacs, J.A., S. Vogel, J.M. Albert, J. Fallon, R.T. Davey, R.E. Walker, M.A. Polis, K. Spooner, J.A. Metcalf, M. Baseler, et al. 1996. Controlled trial of interleukin-2 infusions in patients infected with the human immunodeficiency virus. N. Engl. J. Med. 335:1350-1356.

19. Connors, M., J.A Kovacs, S. Krevat, J.C. Gea-Banacloche, M.C. Sneller, M. Flanigan, J.A. Metcalf, R.E. Walker, J. Falloon, M. Baseler, et al. 1997. HIV infection induces changes in CD4+ T-cell phenotype and depletions within the CD4+ T-cell repertoire that are not immediately restored by antiviral or immune-based therapies. Nat. Med. 3:533-540.

20. Centers for Disease Control. 1992. 1993 revised classification system for HIV infection and expanded surveillance case definition for AIDS among adolescents and adults. MMWR (Morb. Mortal. Wkly. Rep.). 4:1-19.

21. Zanussi, S., C. Simonelli, M. D'Andrea, C. Caffau, M. Clerici, U. Tirelli, and P. De Paoli. 1996. CD8+ lymphocyte phenotype and cytokine production in long-term non-progressor and in progressor patients with HIV-1 infection. Clin. Exp. Immunol. 105:220-224.

22. Zanussi, S., C. Simonelli, M. D’Andrea, M. Comar, E. Bidoli, M. Giacca, U. Tirelli, E. Vaccher, and P. De Paoli. 1996. The effects of antineoplastic chemotherapy on HIV disease. AIDS Res. Hum. Retroviruses. 12:1703-1707.

23. Comar, M., C. Simonelli, S. Zanussi, P. De Paoli, E. Vaccher, U. Tirelli, and M. Giacca. 1997. Dynamics of HIV mRNA expression in patients with long-term non-progressive HIV-1 infection. J. Clin. Invest. 100:893-903.

24. Armitage, P., and G. Berry. 1987. Statistical methods in medical research. Blackwell Scientific Publications, Oxford, UK

25. Helbert, M.R., J. L'age-Stehr, and N.A. Mitchison. 1993. Antigen presentation, loss of immunological memory and AIDS. Immunol. Today. 14:340-344.
26. Benito, J.B., J.M. Zabay, J. Gil, M. Bermejo, A. Escudero, E. Sanchez, and E. Fernandez-Cruz. 1997. Quantitative alterations of the functionally distinct subsets of CD4 and CD8 T lymphocytes in asymptomatic HIV infection changes in the expression of CD45RO, CD45RA, CD11b, CD38, HLA-DR and CD25 antigens. J. Acquired Immune Defic. Syndr. Hum. Retrovirol. 14:128-135.

27. Zanussi, S., M. D'Andrea, C. Simonelli, U. Tirelli, and P. De Paoli. 1996. Serum levels of RANTES and MIP-1 in HIV-positive long-term survivors and progressor patients. AIDS (Lond.). 10:1450-1451.

28. Paxton, W.A., S.R. Martin, D. Tse, T.R. O'Brien, J. Skurick, N.L. Van Devanter, N. Padian, J.F. Braun, D.P. Dotler, S.N. Wolinsky, and R.A. Koup. 1996. Relative resistence to HIV-1 infection of CD4 lymphocytes from persons who remain uninfected despite multiple high-risk sexual exposures. Nat. Med 2:412-417.

29. Urba, W.J., R.G. Steis, D.L. Longo, W.C. Kopp, A.E. Maluish, L. Marcon, D.L. Nelson, H.C. Stevenson, and J.W. Clark. 1990. Immunomodulatory properties and toxicity of interleukin 2 in patients with cancer. Cancer Res. 50: 185-192.

30. Schaafsma, M.R., J.H.F. Falkenburg, J.E. Landegent, N. Duinkerken, S Osanto, P. Ralph, K. Kaushansky, G. Wagemaker, J. Van Damme, R. Willemze, and W.E. Fibbe. 1991. In vivo production of interleukin-5, granulocytemacrophage colony-stimulating factor, macrophage colony-stimulating factor, and interleukin-6 during intravenous administration of high-dose interleukin-2 in cancer patients. Blood. 78:1981-1987.

31. Davey, R.T., Jr., D.G. Chaitt, S.C. Piscitelli, M. Wells, J.A. Kovacs, R.E. Walker, J. Falloon, M.A. Polis, J.A. Metcalf, H. Masur, et al. 1997. Subcutaneous administration of interleukin-2 in human immunodeficiency virus type 1-infected persons. J. Infect. Dis. 175:781-789.

32. Kovacs, J.A., M. Baseler, R.J. Dewar, S. Vogel, R.T. Davey, J. Falloon, M.A. Polis, R.E. Walker, R. Stevens, N.P. Salzman, J.A. Metcalf, H. Masur, and H.C. Lane. 1995. Increases in CD4 T lymphocytes with intermittent courses of interleukin-2 in patients with human immunodeficiency virus infection. A preliminary study. N. Engl. J. Med. 332:567-575.

33. Vanham, G., L. Kestens, I. De Meester, J. Vingerhoets, G. Penne, G Vanhoof, S. Scharpè, H. Heyligen, E. Bosmans, J.L. Ceuppens, and P. Gigase. 1993. Decreased expression of the memory marker CD26 on both CD4+ and CD8+ T lymphocytes of HIV-infected subjects. J. Acquired Immune Defic. Syndr. 6:749-757.

34. Meyaard, L., S.A. Otto, B. Hooibrink, and F. Miedema. 1994. Quantitative analysis of CD4+ T cell function in the course of human immunodeficiency virus infection. Gradual decline of both naive and memory alloreactive T cells. J. Clin. Invest. 94:1947-1952.

35. Bell, E.B., and S.M. Sparshott. 1990. Interconversion of CD45R subsets of CD4 T cells in vivo. Nature. 348:163-166.

36. Roth, M.D. 1994. Interleukin 2 induces the expression of CD45RO and the memory phenotype by CD45RA+ peripheral blood lymphocytes. J. Exp. Med. 179:857-864.

37. Janossy, G., N. Borthwick, R. Lomnitzer, E. Medina, S.B. Squire, A.N. Phillips, M. Lipman, M.A. Johnson, C. Lee, and M. Bofill. 1993. Lymphocyte activation in HIV-1 infection. I. Predominant proliferative defects among CD45RO+ cells of the CD4 and CD8 lineages. AIDS (Lond.). 7:613-624.

38. Weng, N., B.L. Levine, C.H. June, and R.J. Rhodes. 1995. Human naive and memory $\mathrm{T}$ lymphocytes differ in telomeric length and replicative potential. Proc. Natl. Acad. Sci. USA. 92:11091-11094.

39. Woods, T.C, B.D. Roberts, S.T. Butera, and T.M. Folks. 1997. Loss of inducible virus in CD45RA naive cells after human immunodeficiency virus-1 entry accounts for preferential viral replication in CD45RO memory cells. Blood. 89:1635-1641.

40. Ben-Sasson, S.Z., G. Le Gros, D.H. Conrad, F.D. Finkelman, and W.A Paul. 1990. IL-4 production by T cells from naive donors. IL-2 is required for IL-4 production. J. Immunol. 145:1127-1136.

41. Schell-Toeller, D., E. Richter, K.M. Toellner, N. Reiling, H.H. Wacker, H.D. Flad, and J. Gerdes. 1995. CD26 expression in leprosy and other granulomatous diseases correlates with the production of interferon- $\gamma$. Lab. Invest. 73 : 685-691.

42. Sakito, S., Y. Ueki, K. Eguchi, Y. Kawabe, and S. Nagataki. 1995. Serum cytokines in patients with rheumatoid arthritis. Correlation of interferon and tumor necrosis factor with the characteristics of peripheral blood mononuclear cells. Rheumatol. Int. 15:31-37.

43. Pizzolo, G., F. Vinante, L. Morosato, G. Nadali, M. Chilosi, G. Gandini, A. Sinicco, R. Raiteri, G. Semenzato, H. Stein, and G. Perona. 1994. High serum level of the soluble form of CD30 molecule in the early phase of HIV-1 in fection as an independent predictor of progression to AIDS. AIDS (Lond.). 8: $741-745$.

44. Romagnani., S. 1996. TH1 and TH2 in human diseases. Clin. Immunol. Immunopathol. 80:225-235.

45. Cook, D.N., M.A. Beck, T.M. Coffman, S.L. Kirby, J.F. Sheridan, I.B. Pragnell, and O. Smithies. 1995. Requirement of MIP1 for an inflammatory response to viral infection. Science. 269:1583-1585. 